\title{
Factors Affecting Interindividual Variability of Hepatic UGT2B17 Protein Expression Examined Using a Novel Specific Monoclonal Antibody ${ }^{\mathbb{}}$
}

\author{
Jean-Philippe Émond, Adrien Labriet, Sylvie Desjardins, Michèle Rouleau, Lyne Villeneuve, \\ Hélène Hovington, Hervé Brisson, Louis Lacombe, David Simonyan, Patrick Caron, \\ Martine Périgny, Bernard Têtu, John K. Fallon, Kathrin Klein, Philip C. Smith, Ulrich M. Zanger, \\ Chantal Guillemette, and Eric Lévesque
}

Centre Hospitalier Universitaire (CHU) de Québec Research Centre and Faculty of Medicine (J.-P.É., S.D., H.H., H.B., L.L., M.P., B.T., E.L.) and CHU de Québec Research Centre and Faculty of Pharmacy, Laval University (A.L., M.R., L.V., P.C., C.G.), and Statistical and Clinical Research Platform, CHU de Québec Research Centre (D.S.), Québec, Canada.); Eshelman School of Pharmacy, University of North Carolina, Chapel Hill, North Carolina (J.K.F., P.C.S.); and Dr. Margarete Fischer-Bosch Institute of Clinical Pharmacology, Stuttgart, and University of Tübingen, Tübingen, Germany (K.K., U.M.Z.)

Received January 9, 2019; accepted February 21, 2019

\section{ABSTRACT}

Accurate quantification of the metabolic enzyme uridine diphosphoglucuronosyltransferase (UGT) UGT2B17 has been hampered by the high sequence identity with other UGT2B enzymes (as high as $94 \%$ ) and by the lack of a specific antibody. Knowing the significance of the UGT2B17 pathway in drug and hormone metabolism and cancer, we developed a specific monoclonal antibody (EL-2B17mAb), initially validated by the lack of detection in liver microsomes of an individual carrying no UGT2B17 gene copy and in supersomes expressing UGT2B enzymes. Immunohistochemical detection in livers revealed strong labeling of bile ducts and variable labeling of hepatocytes. Expression levels assessed by immunoblotting were highly correlated to mass spectrometry-based quantification ( $r=0.93$ ), and three major expression patterns (absent, low, or high) were evidenced. Livers with very low expression were carriers of the functional rs59678213 G variant, located in the binding site for the transcription factor forkhead box A1 (FOXA1) of the UGT2B17 promoter. The highest level of expression was observed for individuals carrying at least one rs59678213 A allele. Multiple regression analysis indicated that the number of gene copies explained only $8 \%$ of UGT2B17 protein expression, $49 \%$ when adding rs59678213, reaching $54 \%$ when including sex. The novel EL-2B17mAb antibody allowed specific UGT2B17 quantification and exposed different patterns of hepatic expression. It further suggests that FOXA1 is a key driver of UGT2B17 expression in the liver. The availability of this molecular tool will help characterize the UGT2B17 level in various disease states and establish more precisely the contribution of the UGT2B17 enzyme to drug and hormone metabolism.

\section{Introduction}

Uridine diphospho-glucuronosyltransferases (UGTs) are key enzymes that act at multiple levels in the human body, including in the liver, kidneys, intestines, prostate, and multiple other tissues (Beaulieu et al., 1996; Turgeon et al., 2001; Guillemette et al., 2014; Stingl et al., 2014; Margaillan et al., 2015a). This metabolic pathway inactivates many commonly used drugs, such as statins, antidepressants, and antineoplastic agents (Balliet et al., 2009; Kang et al., 2010; Guillemette et al., 2014;

This research was funded by the CHU de Québec (to E.L.) by the Canadian Institutes of Health Research (FRN-42392 and FRN-152986). E.L. is recipient of the CIHR phase 2 clinician-scientist award. C.G. holds a Canada Research Chair in Pharmacogenomics. A.L. receives graduate scholarships from the Fonds d'enseignement et de recherche of the Faculty of Pharmacy and the Fonds de Recherche du Québec-Santé.

https://doi.org/10.1124/dmd.119.086330.

S This article has supplemental material available at dmd.aspetjournals.org.
Stingl et al., 2014). Of the 19 human UGT enzymes, UGT2B17 specifically metabolizes and inactivates sex-steroid hormones, such as testosterone and dihydrotestosterone in the prostate (Beaulieu et al., 1996). UGT2B17 activity is highly variable in tissues such as the liver (Lazarus et al., 2005; McCarroll et al., 2006; Liu et al., 2014; Margaillan et al., 2015b; Bhatt et al., 2018); therefore, a better understanding of the determinants of UGT2B17 protein expression could help predict its influence on xenobiotics and endobiotics under both normal conditions and disease states. To date, quantification of UGT2B17 has been hampered by the high sequence identity with other UGT2B subfamily members. For instance, UGT2B15 shares more than $94 \%$ of its amino acid sequence with UGT2B17 (Beaulieu et al., 1996). This explains, at least in part, the current lack of a specific antibody that does not crossreact with any of the other UGT2B members, namely, UGT2B4, 2B7, 2B10, 2B11, 2B15, and 2B28 (Guillemette et al., 2010, 2014). Thus, quantification of UGT2B17 has been extrapolated mostly from correlative studies of RNA expression and enzymatic activities (Lazarus et al., 2005; Izukawa et al., 2009; Chen et al., 2010; Jones and Lazarus, 2014;

ABBREVIATIONS: CNV, copy number variation; FOXA1, forkhead box protein A1; HepG2, hepatocellular carcinoma cell line G2; IHC, immunhistochemistry; LAPC4, prostate cancer xenograft cells; LNCaP, lymph node carcinoma of the prostate; MS, mass spectrometry; PCR, polymerase chain reaction; SNP, single-nucleotide polymorphism; UGT, uridine diphospho-glucuronosyltransferase; WB, Western blot. 
Liu et al., 2014). These approaches have limitations, especially in the context of splicing mechanisms and overlapping substrate specificities (Hum et al., 1999; Guillemette et al., 2014; Tourancheau et al., 2016, 2018).

More recently, mass spectrometry (MS)-based quantification of UGT2B17 was developed using signature peptides (Fallon et al., 2013; Margaillan et al., 2015b; Bhatt et al., 2018). A study based on MS quantification showed that $26 \%$ of the variability of hepatic UGT2B17 protein expression could be explained using a model thay considers age, sex, and five genetic variations [i.e., rs7436962, rs9996186, rs28374727, rs4860305, and gene copy number variation (CNV)] (Bhatt et al., 2018). It is thus likely that additional molecular determinants exist, as most of this variability remains unexplained. One single nucleotide variation (SNP) in the UGT2B17 gene rs59678213, located in a forkhead box protein A1 (FOXA1) binding site in the promoter region, influenced $U G T 2 B 17$ gene expression in prostate cancer cells (Hu et al., 2010), but its impact on expression in human tissues remains to be elucidated. Another study has suggested that rs6817882, also located in the $U G T 2 B 17$ promoter region, could be of interest (Liu et al., 2014). This marker is in strong linkage disequilibrium with rs59678213 and was correlated with RNA levels in the liver (Liu et al., 2014); however, the impact of these SNPs on UGT2B17 protein expression was not quantified in these two studies (Hu et al., 2010; Liu et al., 2014).

The first objective of this study was to develop a specific monoclonal antibody for more accurate quantification of the UGT2B17 enzyme in human tissues. The second objective was to evaluate the heterogeneity in UGT2B17 protein expression observed in the human liver and gain further insights into molecular determinants of its expression. A specific antibody would be an additional tool for the scientific community to quantify the UGT2B17 enzyme and expedite study of this important pathway involved in major metabolic functions.

\section{Materials and Methods}

Human Tissue Specimens. Supersomes overexpressing human UGT2B enzymes were purchased from Corning Life Sciences (Woburn, MA). Microsomes from pooled human liver (HLM), intestine (HIM), and kidney were purchased from Xenotech (Lexena, KS). Forty-eight human liver specimens from white healthy donors, including an equal number of men and women, were available for this study. The characteristics of the donors have been described previously (Sumida et al., 1999; Gomes et al., 2009). Paraffin-embedded normal liver samples were available from four additional individuals. Written consent was obtained from donors, and the study was approved by the ethics committee of the CHU de Québec, Laval University [no. 2012-245; 5.7.02.05 (34.05.08)].

Production of the UGT2B17 Protein Monoclonal Antibody. The monoclonal antibody directed against the UGT2B17 protein was produced using the custom monoclonal service at GenScript (Piscataway, NJ) according to their proprietary approach. An immunogenic peptide 83-KNDLEDFFMKMFDRWTY-99 (Supplemental Fig. 1) was selected based on immunogenicity and specificity relative to the sequence of other human proteins, including the six other UGT2Bs. Clone no. 8B7E3-1 was used to produce the final antibody named EL-2B17mAb. Hybridoma cells were grown in low $\mathrm{IgG}$ bovine serum, and the monoclonal antibody was purified from the culture medium by protein A/G affinity chromatography.

UGT2B17 mRNA and Protein Expression. UGT2B17 mRNA expression levels were assessed in 11 livers for which mRNA was available, as previously described (Chouinard et al., 2006; Lepine et al., 2010), using $36 B 4$ as an internal amplification standard. The location of the primers is displayed in Supplemental Fig. 1. Quantification of hepatic UGT2B17 by targeted proteomics for 48 livers with the signature peptide shown in Supplemental Fig. 1 was previously reported (Margaillan et al., 2015b). The lower level of quantification was set at $0.2 \mathrm{pmol} / \mathrm{mg}$ protein. Quantification of hepatic UGT2B17 by Western blot (WB) was performed for 32 individual liver samples using available microsomal proteins. Supersomes $(0.5 \mu \mathrm{g})$ and microsomes (2 or $10 \mu \mathrm{g})$ were mixed with Laemmli sample buffer (Bio-Rad Laboratories, Hercules, CA), heated at $100^{\circ} \mathrm{C}$ for 5 minutes, separated on a $10 \%$ SDS-PAGE gel, and transferred to a nitrocellulose membrane. The membranes were blocked with phosphate-buffered saline containing 0.2\% Igepal (Sigma Aldrich, St. Louis, MO) and 5\% dry milk and then probed with the EL-2B17mAb monoclonal antibody (1:2000) overnight using standard protocols. The pan-UGT2B EL-93 polyclonal antibody (1:2000) was also used as described in a previous study (Levesque et al., 1997).

Immunohistochemistry. Sections $(5 \mu \mathrm{m})$ of paraffin-embedded normal liver samples were deparaffinized, rehydrated, and processed using a PT Link system (Agilent Technologies, Mississauga, ON). Heat-induced epitope retrieval was achieved using the EnVision FLEX Target Retrieval Solution, Low pH (Agilent), and tissues were stained with EL-2B17mAb (1:1000) using the IDetect SuperStain horseradish peroxidase polymer kit (Empire Genomics, Buffalo, NY) according to the manufacturer's instructions.

UGT2B17 Genetic Status. Genotypes were assessed for the 48 individuals using available material consisting of DNA isolated from human liver specimens. CNV of the UGT2B17 gene was determined as described previously (McCarroll et al., 2006; Menard et al., 2009). For rs59678213, a PCR amplicon spanning the promoter region was sequenced to assess the genotype. The amplification was performed in $25-\mu 1$ reactions containing $50 \mathrm{ng}$ genomic DNA, $0.2 \mathrm{mM}$ each dNTP deoxynucleotide solution mix of dATP, dCTP, dGTP, and dTTP), $3 \mathrm{mM}$ $\mathrm{MgCl}_{2}, 0.3 \mathrm{mM}$ each of the two primers (F: 5'-CCTCTCACCTGCCACTGTTC-3' and R: 5'-CATCTGCCAGAAGGACATCAAATT-3') and 1.25 units AmpliTaq Gold 360 DNA Polymerase (Thermo Fisher Scientific, Waltham, MA). The reaction was incubated at $95^{\circ} \mathrm{C}$ for 10 minutes, followed by 35 cycles of $95^{\circ} \mathrm{C}$ for 30 seconds, $62^{\circ} \mathrm{C}$ for 40 seconds, and $72^{\circ} \mathrm{C}$ for 30 seconds, with a final elongation step at $72^{\circ} \mathrm{C}$ for 7 minutes. Sanger sequencing was done using an ABI 3700 automated sequencer (Applied Biosystems, Foster City, CA). Allele frequency of the rs59678213 and rs6817882 variants and calculation of their linkage disequilibrium among ethnic groups from the 1000 Genomes Project phase 3 were obtained through the Ensembl genome browser release 94, October 2018, assembly GRCh37.p13, accessed on November 26, 2018. CNV of the UGT2B17 gene in the same ethnic groups were obtained from Xue et al. (2008).

Reporter Gene Assays. The human UGT2B17 DNA promoter construct $(-2413$ to +1$)$ was isolated as described in a previous study (Beaulieu et al., 1997). Using this construct as the template, we generated a construct containing guanine at position -155 (corresponding to rs59678213) using Q5 Site-Directed Mutagenesis kit reagents (New England Biolabs, Ipswich, MA) and primers 5'-GAGTAATTGTgAATATAAAAGAACACC-3' (forward) and 5'TAGTCAAGCAATAATTTTTATGAC- $3^{\prime}$ (reverse). Mutagenesis of these constructs was performed to generate an adenine at position -2033 (corresponding to rs6817882) using primers 5'-ATTTTATATTaTTTTATGCTCAGTACCTG-3' (forward) and 5'-AATCTGAGAGGGTCCTTC-3' (reverse). The prostate cancer LNCaP (lymph node carcinoma of the prostate) and the hepatocellular carcinoma (HepG2) cell lines were purchased from the American Type Culture Collection (Manassas, VA). The HuH7 cell line was obtained from the Japanese Collection of Research Bioresources Cell Bank (Tokyo, Japan), and the prostate cancer cell line LAPC4 (prostate cancer xenograft cells) was a gift from Dr. F. Pouliot (CHU de Québec Research Centre, Laval University, QC). Cells were maintained in RPMI-1640 medium (LNCaP) or Dulbecco's modified Eagle's medium (LAPC4, HepG2, HuH7) supplemented with $10 \%$ (v/v) fetal bovine serum and antibiotics (100 IU/ml penicillin and $100 \mathrm{pg} / \mathrm{ml}$ streptomycin). RPMI-1640 for LNCaP culture was also supplemented with $2 \mathrm{mM}$ glutamine. Culture medium components were all from Wisent Bioproducts (St. Bruno, QC). All transfections were performed using LipofectAMINE 2000 (Invitrogen, Carlsbad, CA) and OptiMEM Reduced-Serum Medium (Life Technologies, Burlington, ON). Cells were plated into 24 -well plates at a density of $1 \times 10^{5}(\mathrm{HepG} 2$ and $\mathrm{HuH} 7), 8.0 \times$ $10^{4}(\mathrm{LNCaP})$, or $2 \times 10^{5}$ (LAPC4) cells/well. For the luciferase assays, cells were transfected 24 hours after plating with 200 (HepG2 and $\mathrm{HuH} 7$ ) or $500 \mathrm{ng}$ (LNCaP and LAPC4) of the appropriate constructs and 20 (HepG2 and $\mathrm{HuH} 7$ ) or $50 \mathrm{ng}$ (LNCaP and LAPC4) of the Renilla luciferase construct (internal control). Cells were lysed 48 hours after transfection and assessed for luciferase activity using Dual-Luciferase Reporter Assay kit reagents (Promega, Madison, WI).

Statistical Analysis. Differences in UGT2B17 expression among genetic groups were confirmed using the Kruskal-Wallis test, and Wilcoxon MannWhitney test was used for sex. Correlation analysis was performed by the Spearman rank method. A multiple regression analysis was performed to evaluate the associations between UGT2B17 protein expression, sex, and 
TABLE 1

Protein sequence alignment of the antigen peptide of uridine diphospho-glucuronosyltransferase (UGT) 2 B17 with the corresponding sequence of related UGT2B family members

Amino acid sequences of other human UGT2B corresponding to UGT2B17 residues 83-99, used as the antigen for the antibody production, are shown. Amino acids that are identical to UGT2B17 are indicated in bold. The sequence alignment was performed using Clustal Omega (https://www.ebi.ac.uk/Tools/msa/clustalo/).

\begin{tabular}{|c|c|c|c|c|}
\hline \multirow{2}{*}{ UGT Proteins } & \multirow{2}{*}{ Accession No. } & \multirow{2}{*}{ Sequence } & \multicolumn{2}{|c|}{ Identity with UGT2B17 (\%) } \\
\hline & & & Antigen Region & Full-Length \\
\hline UGT2B17 & O75795 & ${ }_{83} \mathrm{KNDLEDFFMKMFDRWTY}_{99}$ & & \\
\hline UGT2B4 & P06133 & ${ }_{83}$ KTEFEDIIKQLVKRWAE 99 & 29.4 & 78.1 \\
\hline UGT2B7 & P16662 & ${ }_{83}$ KTELENFIMQQIKRWSD 99 & 41.2 & 76.8 \\
\hline UGT2B10 & P36537 & ${ }_{82}$ KTEFENIIMQLVKRLSE 98 & 23.5 & 76.8 \\
\hline UGT2B11 & O75310 & ${ }_{83} \mathbf{K T E F E N I I M Q Q V K R W S D}{ }_{99}$ & 29.4 & 76.6 \\
\hline UGT2B15 & P54855 & ${ }_{83}$ KNYLEDSLLKILDRWIY $_{99}$ & 58.8 & 94.2 \\
\hline UGT2B28 & Q9BY64 & ${ }_{83}$ KTEFENIIMQQVKRWSD 99 & 29.4 & 75.3 \\
\hline
\end{tabular}

genotype. Differences between constructs in luciferase assays were tested using a two-tailed Student's $t$ test. $P<0.05$ was considered statistically significant. Analyses were corrected for the $\alpha$ error using the Bonferroni adjustment method. All statistical analyses were performed with the software SAS 9.4 by SAS Institute Inc. (Cary, NC).

\section{Results}

EL-2B17mAb is a Specific Human UGT2B17 Monoclonal Antibody. The peptide sequence 83-KNDLEDFFMKMFDRWTY-99, unique to UGT2B17 among human UGT2B proteins (Supplemental Fig. 1; Table 1), was chosen to produce the mouse monoclonal EL-2B17mAb antibody. The specificity of EL-2B17mAb was initially evaluated using supersomes overexpressing individual human UGT2B proteins. A protein band was detected only in the UGT2B17 supersomes at the expected molecular weight of $55 \mathrm{kDa}$ (Fig. 1A). The unique reactivity with the UGT2B17 protein was further confirmed with additional recombinant UGT2B enzymes, namely, UGT2B11 and UGT2B28, not commercially available as supersomes (Supplemental Fig. 2). The antibody was subsequently tested in major drugmetabolizing tissues, displaying a strong signal in the liver and intestine and none in the kidney, consistent with the known tissue distribution of UGT2B17 (Fig. 1B). Lastly, no signal was found in a UGT2B17deficient HLM from an individual carrying no UGT2B17 gene copy, proficient in the expression of other UGT2B enzymes (not shown), confirming the identity of the detected protein band as UGT2B17 and the specificity of the UGT2B17mAb (Fig. 1C). Normal liver tissues from four individuals labeled with EL-2B17mAb by immunohistochemistry (IHC) revealed a strong labeling of bile duct epithelial cells (Fig. 2; Supplemental Fig. 3). By contrast, a widely variable labeling intensity was noted for hepatocytes among liver samples and within each sample. Hepatocytes of the periportal zones were more intensely labeled and displayed a preferential nuclear labeling, whereas hepatocytes in the centrilobular regions were weakly labeled. The intensity of cytoplasmic staining was variable, from strong to moderate in liver $\mathrm{A}$, which displayed strongest overall labeling (Fig. 2), to weak or undetected in the three other livers (Supplemental Fig. 3). Initial experiments also suggest that the antibody does not impair UGT2B17 activity in microsomes (data not shown).

Gene Deletion and the Genetic Variant rs59678213 Largely Contribute to the Highly Variable Hepatic UGT2B17 Expression. The relative UGT2B17 protein expression levels assessed by WB in HLM samples was highly variable $(\mathrm{CV}=118 \%)$ and correlated with the values obtained by quantitative real-time MS for these same samples $(r=0.93 ; P<0.0001)$ (Fig. 3). A significant correlation with $U G T 2 B 17$ mRNA expression was also observed with protein abundance assessed by MS $(r=0.70, P=0.038)$ and WB $(r=0.75, P=0.009)$ for a subset of tested livers for which mRNA was available (not shown). According to UGT2B17 CNV and based on quantification data, samples were categorized into three groups corresponding to no gene copy $(n=9)$, one gene copy $(n=21)$, and two gene copies $(n=18)$ (Fig. 4). As expected,
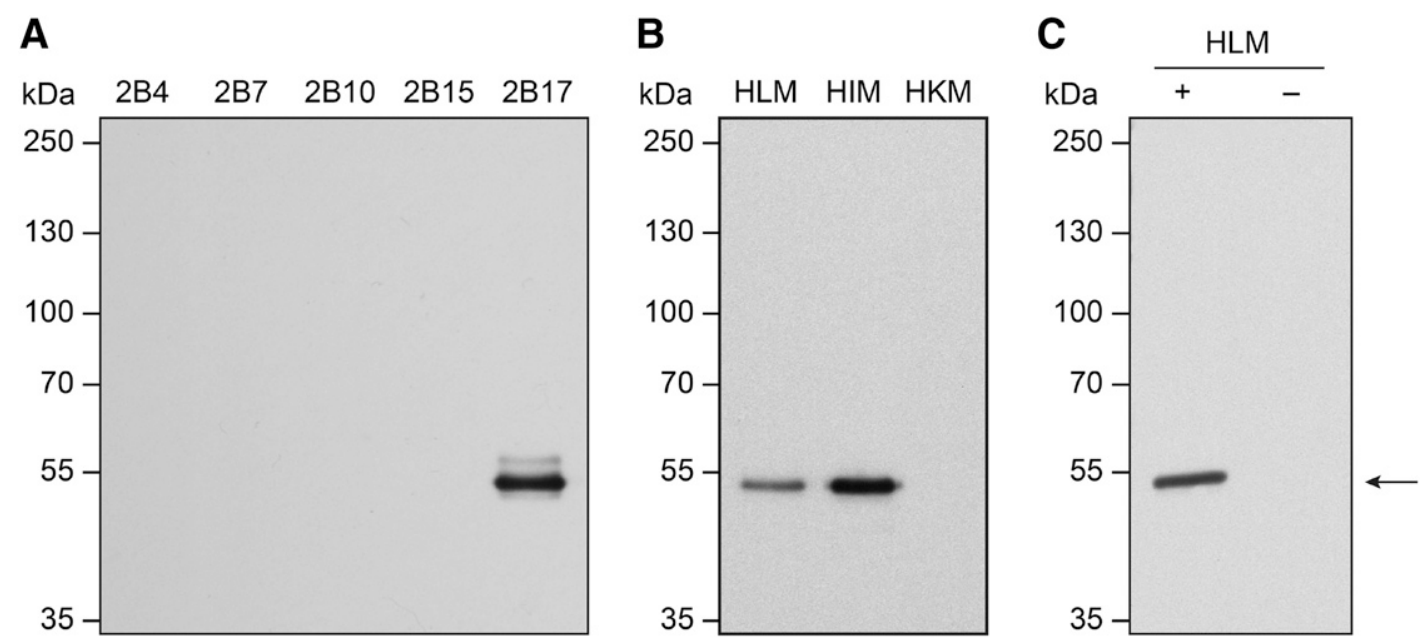

Fig. 1. Specificity of the novel monoclonal antibody EL-2B17mAb directed against the UGT2B17 protein. (A) Recombinant human UGT2Bs in supersomes (0.5 $\mu \mathrm{g}$ ). (B) Pooled HLMs, HIMs, and human kidney microsomes (HKMs) $(10 \mu \mathrm{g})$. (C) Pooled HLMs $(2 \mathrm{~B} 17+)$ and liver microsomes from an individual carrying no UGT2B17 gene copy $(2 \mathrm{~B} 17-)$. The positions of molecular weight markers are given on the left. Arrow indicates the molecular weight of UGTs. 

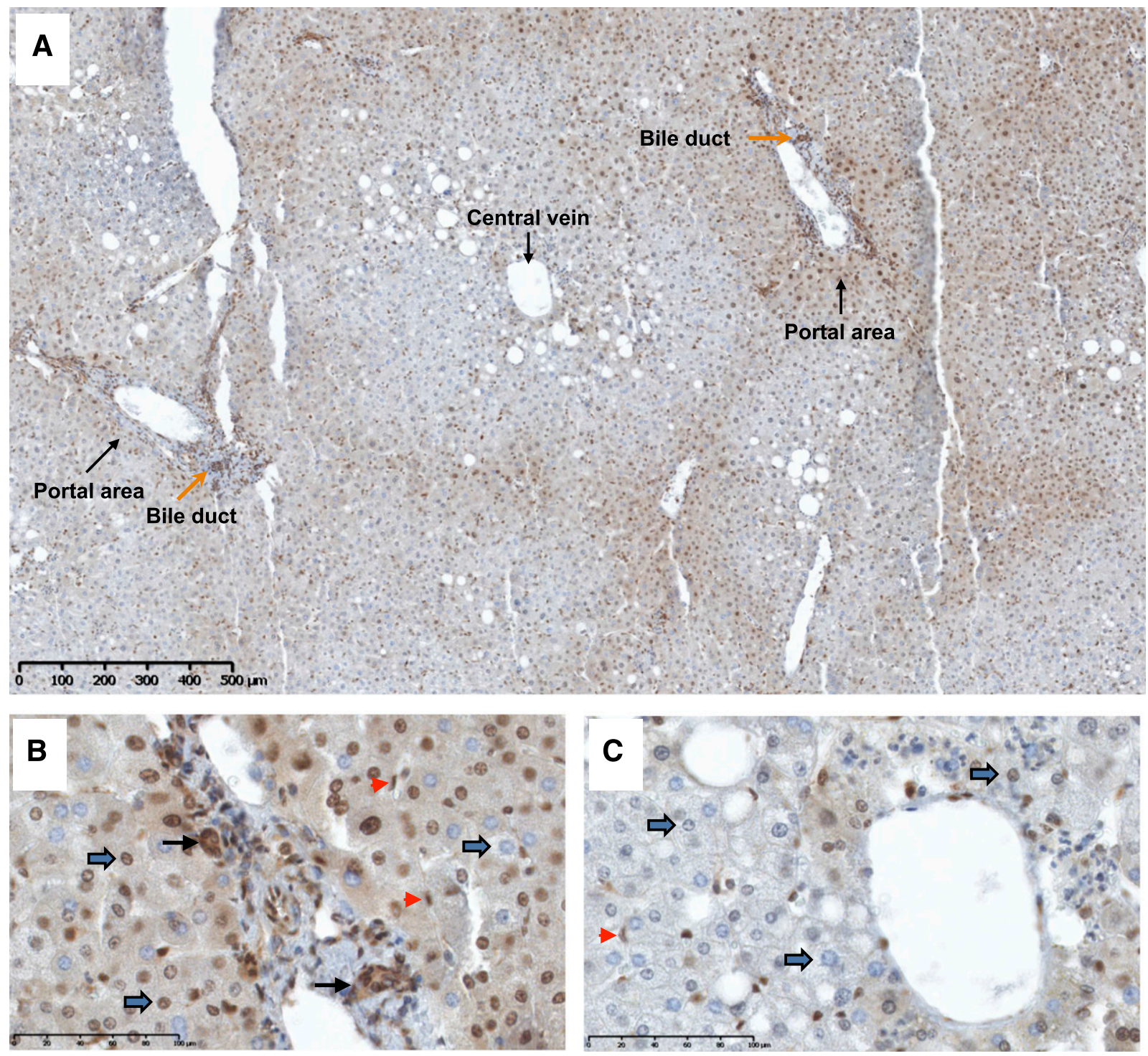

Fig. 2. Immunohistochemical detection of UGT2B17 with EL-2B17mAb in a normal human liver. (A) Overview of labeling distribution. Bile ducts (orange arrows) are strongly labeled, whereas staining intensity of hepatocytes in the portal and central vein areas is variable. Scale bar, $500 \mu \mathrm{m}$. (B) Enlarged view of a periportal area. Nuclei and cytoplasm of bile duct epithelial cells (thin black arrows) are strongly stained. Hepatocytes (large blue arrows) are variably stained, with a predominant nuclear labeling. Inflammatory cells of sinusoidal spaces are also labeled (red arrows). (C) Enlarged view of the central vein area showing a weaker labeling of hepatocytes (large blue arrows). In (B and C), bars represent $100 \mu \mathrm{m}$. The liver shown corresponds to liver A in Supplemental Fig. 3.

the UGT2B17 protein was undetected by MS in the group with no gene copy (lower limit of quantification $<0.2 \mathrm{pmol} / \mathrm{mg}$ protein). The median protein abundance in carriers of one gene copy was quite similar to the value observed for those carrying two gene copies $(0.45$ vs. $0.43 ; P=$ 1.0). A significant variability within groups was observed with $156 \%$ $\mathrm{CV}$ and $169 \% \mathrm{CV}$ in carriers of one and two gene copies, respectively. Based on this high variability, not explained by the number of UGT2B17 gene copies, we next determined the association between UGT2B17 protein expression and the occurrence of a functional variation located in the FOXA1 binding sequence of the UGT2B17 promoter, warranted by the frequent occurrence of this genetic variation, including in individuals of European descent (Fig. 5; Supplemental Table 1). Irrespective of the UGT2B17 gene copy number, livers carrying the rs59678213A allele expressed high UGT2B17 levels, whereas all others comprised of only rs59678213G allele showed low expression. This was observed with quantification by both WB (Fig. 6A; Supplemental Figs. 4 and 5) and MS (Fig. 6, B and C; Supplemental Table 2). In a further analysis taking sex into account, female carriers of the rs59678213G allele presented significantly lower UGT2B17 protein levels than did male subjects (median levels of 0.27 vs. $0.32 \mathrm{pmol} / \mathrm{mg}$ protein; $P=0.026$ ), whereas no difference was found between sexes for rs59678213A carriers displaying much higher expression ( $\sim 40$-fold). Multiple regression analysis indicated that at least one gene copy, rs59678213A, and male sex are significantly associated with greater UGT2B17 expression. It further indicated that the number of gene copy explained only $8 \%$ of UGT2B 17 protein expression, $49 \%$ when adding rs59678213, and reached 54\% when including sex $\left(R^{2}=0.54\right.$; multivariate linear regression $)$. Since the UGT2B17 protein is considerably expressed in the prostate, we further tested the reactivity of EL-2B17mAb in prostate cancer cell lines. No expression of UGT2B17 was detected in LAPC4 cells by WB, consistent with the absence of the $U G T 2 B 17$ gene and very low expression in VCaP and 22rv1, homozygous for the rs59678213G allele. In contrast, high expression was observed in LNCaP cells homozygous for the rs59678213A allele (Supplemental Fig. 6). 


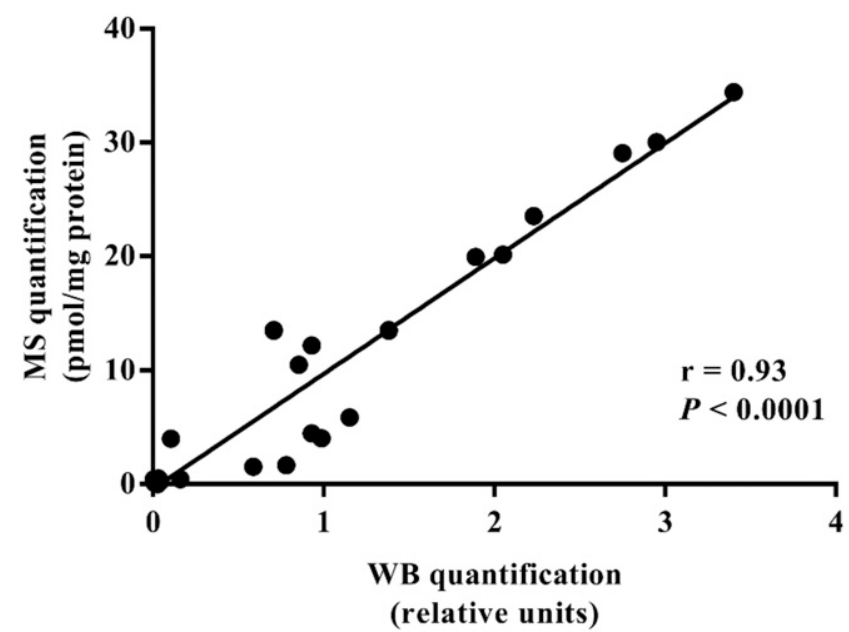

Fig. 3. Correlation between UGT2B17 protein expression in human liver microsomal samples $(n=29)$ determined by MS and immunoblotting (WB) using EL-2B17mAb. Spearman correlation coefficient $(r)$ and corresponding $P$ value are given.

Genetic Variant rs59678213 is a Key Driver of UGT2B17 Expression In Vitro. The variants rs59678213 and rs6817882 significantly affecting $U G T 2 B 17$ mRNA expression were reported to be tightly linked (Hu et al., 2010; Liu et al., 2014); however, similar to the frequency of the $U G T 2 B 17$ gene deletion copy, we observed that their occurrence is highly variable between ethnic groups, ranging from $5 \%$ to $93 \%$ (Fig. 5). Accordingly, we tested the functionality of these two markers using luciferase constructs engineered to reflect all combinations of alleles and tested them in cell models derived from the liver (HepG2 and $\mathrm{HuH7}$ ) and prostate cancer cells carrying the UGT2B17 gene (LNCaP and LAPC4) to identify the functional variant(s). Data supported the functional role of the rs59678213 variant as a major driver of UGT2B17 expression in LNCaP and LAPC4 prostate cancer cells, as well as in hepatic HuH7 cells (Supplemental Fig. 7). No difference in expression was observed in HepG2 cells in the presence of the rs59678213 variant (not shown).

\section{Discussion}

To date, accurate detection and quantification of human UGT2B17 proteins by WB analysis have been hampered by the lack of a highly specific monoclonal antibody. Indeed, the high sequence identity among UGT2B enzymes underlies the technical challenge of developing antibodies with no cross-reactivity with other subfamily members. Despite this very high sequence identity, also observed in the UGT1A subfamily, specific monoclonal antibodies have been developed for UGT1A9 (Oda et al., 2012) and UGT1A10 (Oda et al., 2017) but are lacking for most UGT2B members. Several previous studies have used RNA expression and enzymatic kinetics as a surrogate to protein quantification (Lazarus et al., 2005; Izukawa et al., 2009; Chen et al., 2010; Jones and Lazarus, 2014; Liu et al., 2014). These methods, however, have inherent limitations, such as the presence of extensive alternative splicing events (Tourancheau et al., 2016, 2018) and the intrinsic overlapping substrate specificities characterizing these enzymes (Belanger et al., 1998; Guillemette et al., 2010, 2014). More recently, to circumvent this technical issue related to the absence of highly specific UGT antibodies, MS-based methods were developed to accurately quantify UGT isoforms in human samples using small signature peptides (Sakamoto et al., 2011; Fallon et al., 2013; Sato et al., 2014; Margaillan et al., 2015b; Bhatt et al., 2018).

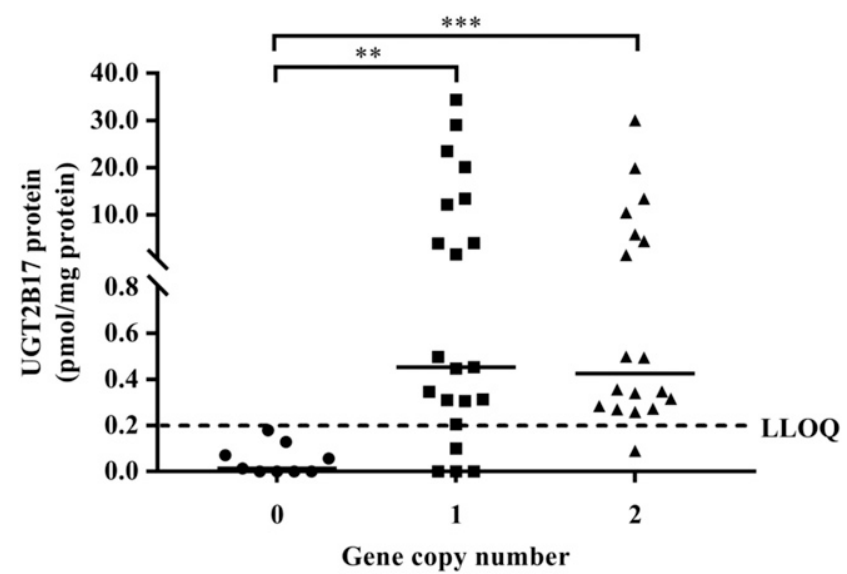

Fig. 4. UGT2B 17 protein abundance in liver samples according to UGT2B17 gene copy number $(n=48)$. Quantification of UGT2B17 protein expression levels was determined by MS. $P$ values were determined using the Kruskal Wallis one-way analysis of variance and corrected by Bonferroni. $* * P<0.01$; *** $P<0.001$.

The specificity of the EL-2B17mAb monoclonal antibody produced in this study is an important additional molecular tool capable of specifically detecting the UGT2B17 protein both in WB and IHC and could expedite clinical advances on the significance of UGT2B17 in drug metabolism (Turgeon et al., 2003; Kang et al., 2010; Sun et al., 2010; Taghavi et al., 2017; Kahma et al., 2018) and cancer biology (Nadeau et al., 2011; Gruber et al., 2013; Bhoi et al., 2016; Li et al., 2016). It is the first antibody with a unique specificity for UGT2B17 without any cross-reactivity to any other human UGT2B enzymes, including the highly similar UGT2B15. The specificity of the antibody for the protein was conclusively demonstrated by the absence of protein detection in a UGT2B17-deficient liver, the latter expressing other UGT2B enzymes. Moreover, the fact that the correlation between immunoblotting and MS analyses reached $93 \%$ reinforces the notion that both methods are accurate to quantify UGT2B17. The lower degree of correlation between mRNA and protein quantification methods highlights the added value of the more precise assessment of UGT2B17 enzyme in the liver afforded by protein quantification. Quantification by immunoblotting with EL-2B17mAb may constitute a method of choice to assess the 55-kDa canonical UGT2B17 isoform.

Immunohistochemistry of four liver samples further revealed that UGT2B17 is expressed in the nucleus and cytoplasm of bile ducts and hepatocytes located in the periportal zone 1 region, in close contact with blood from the systemic and splanchnic circulations. Quantification of UGT2B17 by immunoblotting in multiple liver samples using EL-2B17mAb strikingly revealed high UGT2B17 expression in nearly half of the specimens, whereas it was undetected or barely detectable in the other half. It led us to evaluate whether the UGT2B17 gene copy number was responsible for this on or off expression pattern. Among the livers tested, 9 of 48 samples had no copy of the gene, explaining the absence of expression in those samples. For all the other samples, the presence of one or two gene copies did not affect protein expression significantly, contrary to our expectations. Although the reasons for this finding are currently not understood, it should be noted that a recent analysis of $C Y P 2 E 1$ copy number variation in liver and lymphocytes led to the conclusion that its expression is gene dosage-insensitive (Tremmel et al., 2016). In contrast, the genotype of the rs59678213 variant located in the FOXA1 binding site of the promoter region of UGT2B17 (Hu et al., 2010) explained a much larger fraction of variability compared with copy number and the A allele was largely associated with the high UGT2B17 expression pattern. This common genetic variation, for which the genotype varies importantly across ethnic 
A

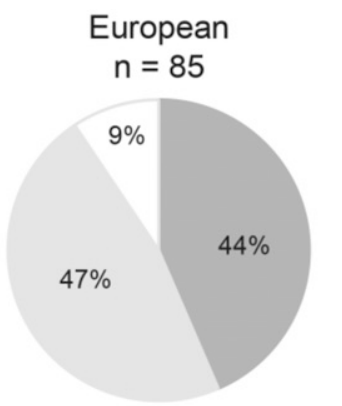

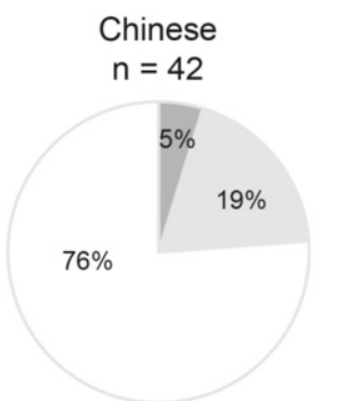
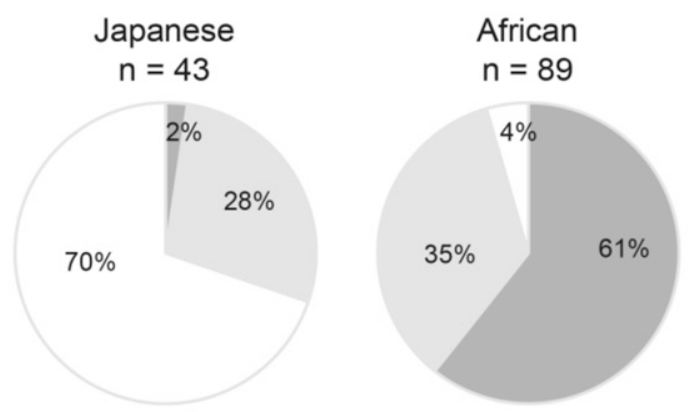

UGT2B17 gene copy number $\square 2 \square 1 \square 0$

B
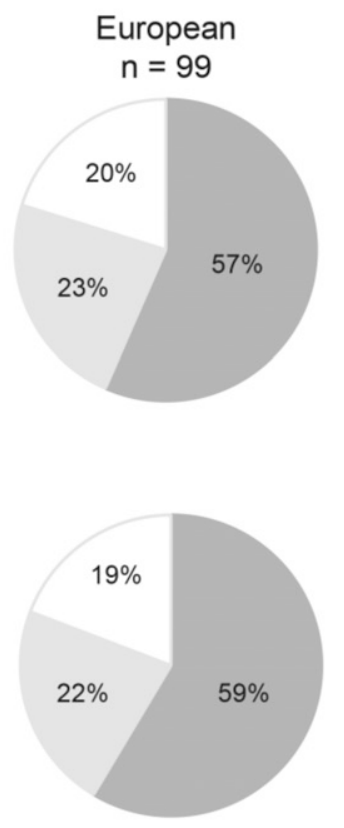

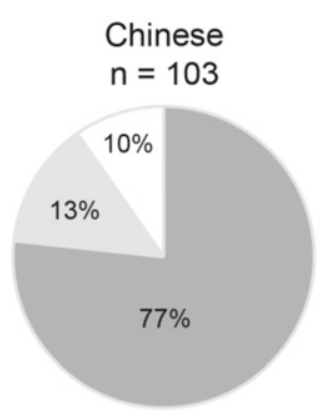

rs59678213

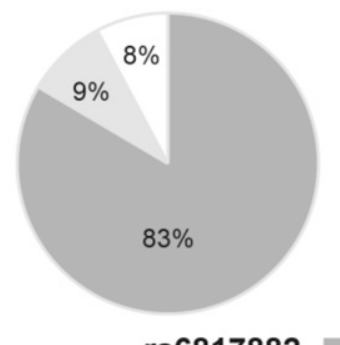

rs6817882
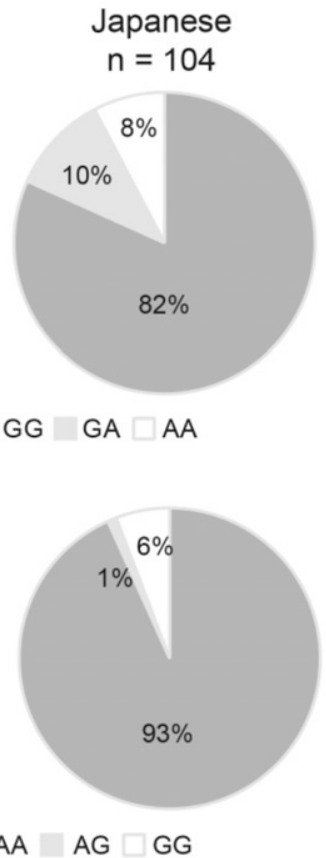
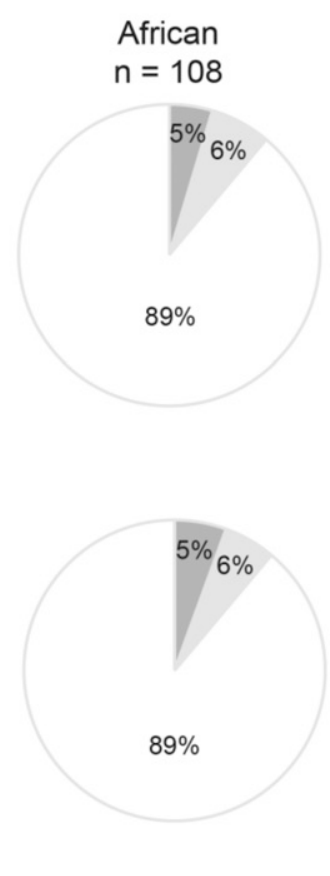

Fig. 5. Frequencies of UGT2B 17 gene variations in diverse ethnic groups. (A) UGT2B17 gene CNV. Frequencies were taken from Xue et al. (2008). (B) Allele frequencies of rs59678213 and rs6817882; (C) Linkage disequilibrium between rs59678213 and rs6817882. For (B and C), data were obtained from the $1000 \mathrm{Genomes}$ phase 3 project. Individuals from European ethnicity correspond to CEU : Utah Residents (CEPH) with Northern and Western European Ancestry, Chinese to CHB : Han Chinese in Beijing, China; Japanese to JPT : Japanese in Tokyo, Japan; and African to YRI : Yoruba in Ibadan, Nigeria.

groups (Fig. 5; Supplemental Table 1), was previously identified to affect FOXA1 binding to the $U G T 2 B 17$ promoter in prostate cancer cells by $\mathrm{Hu}$ et al. (2010). Our functional analysis in liver and prostate cancer cells supported that this genetic variant significantly impacts UGT2B17 expression. It is possible to infer that the rs59678213 explains a significant proportion of the enzyme hepatic expression since it is in strong linkage with rs6817882 in white subjects, which was also associated with a significant variation of UGT2B17 expression in the liver (Liu et al., 2014). Indeed, the high level of expression phenotype observed in WB was systematically associated with the presence of the rs59678213 A variant. On the other hand, the absent or marginal expression phenotype was persistently associated with the rs59678213
$\mathrm{G}$ variant or complete gene deletion. Overall, based on the protein expression data, the inclusion of the UGT2B17 genetic status, including the rs59678213 genetic variant and the CNV, along with sex, as previously reported from mRNA expression data (Gallagher et al., 2010), explains more than $50 \%$ of the variability of UGT2B 17 protein expression in the liver. This knowledge represents an important finding since the current practice essentially considers the gene copy number to predict UGT2B17 expression levels (Gallagher et al., 2007; Wang et al., 2012; Kahma et al., 2018), here demonstrated to reflect only partially UGT2B17 expression. Based on the observed UGT2B17 gene deletion frequency and the occurrence of the rs59678213 G genotype among ethnic groups, our results suggest a high expression of the UGT2B17 
A
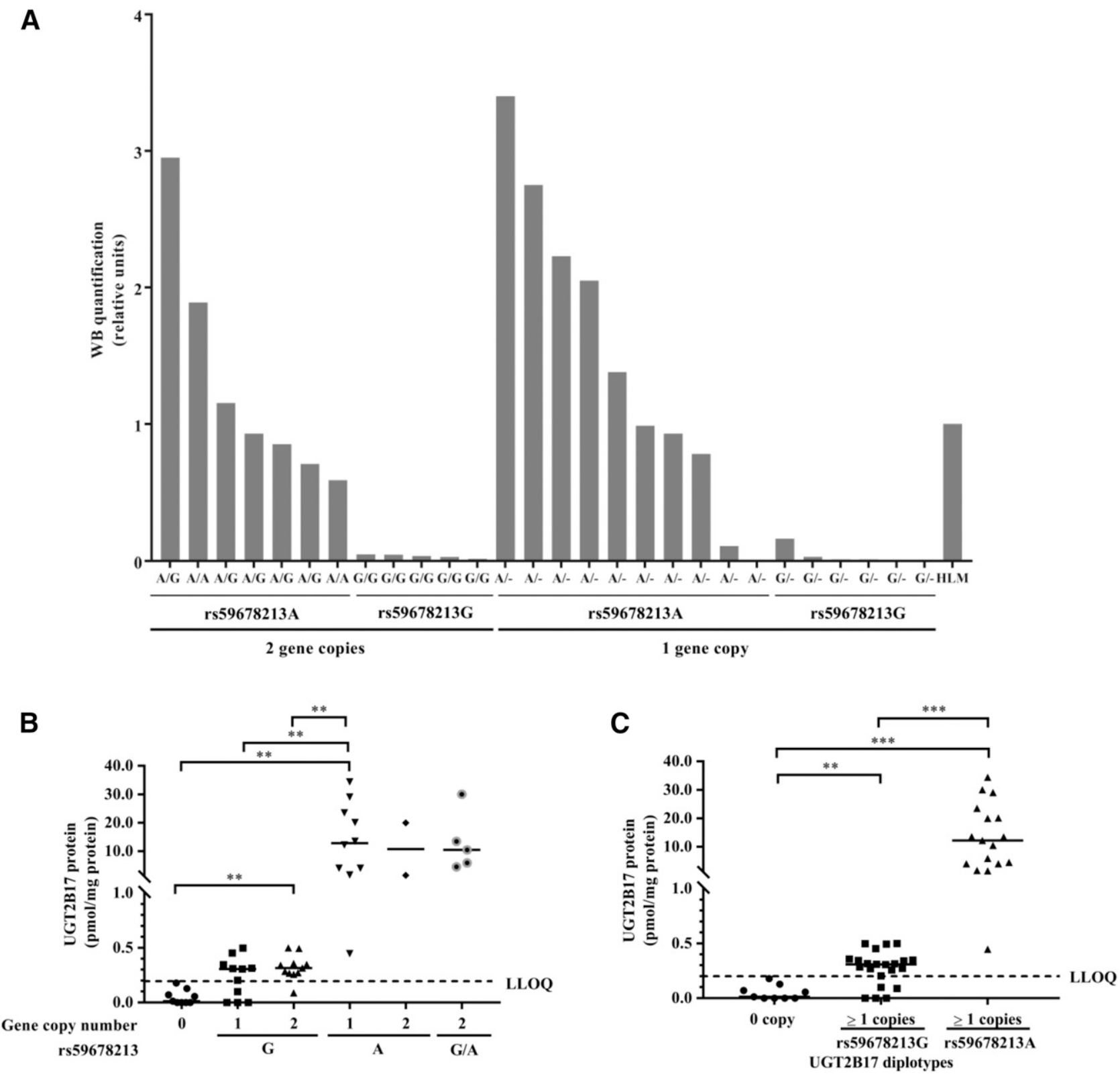

Fig. 6. UGT2B17 protein abundance in human liver is driven by FOXA1 rs59678213A $>$ G status. (A) UGT2B17 expression levels in liver samples $(n=32)$ according to UGT2B17 gene copy number and the rs59678213A $>$ G genotype. UGT2B17 protein levels were as determined by immunoblotting (WB); (B) UGT2B17 protein levels assessed by MS in HLMs $(n=48)$ are stratified by UGT2B17 gene copy number and the rs59678213A $>$ G status; (C) UGT2B17 protein levels as in (B) are grouped according to rs59678213 in individuals with at least one gene copy. Significance was determined using the Kruskal-Wallis one-way analysis of variance and were corrected by Bonferroni. $* * P<0.01 ; * * * P<0.001$.

enzyme in the African population that displays a low deletion frequency and a prevalence of the rs 59678213 A allele of $95 \%$. By contrast, a high expression of UGT2B17 is expected in only $\sim 15 \%$ of Asians (Chinese and Japanese) given the gene deletion frequency and the low prevalence of the rs59678213 A genotype, in only $18 \%-23 \%$ of the Asian population.

Other known determinants of UGT2B17 protein expression were previously described, such as age and additional germline single nucleotide variations (Liu et al., 2014; Bhatt et al., 2018), which we did not test owing to the limited number of liver samples, whereas rs59678213 and rs6817882 were not included in a previous study comprising a much more significant number of liver samples (Bhatt et al., 2018). Based on our observations, it will be critical to test the variation rs59678213 in large cohorts to further our understanding of molecular determinants of UGT2B17 expression.
A major strength of this study is the development of the first monoclonal antibody with unique specificity for UGT2B17 and its validation by the analysis of a UGT2B17-negative liver and through correlation with proteomics quantification. It enables accurate assessment of UGT2B17 by immunoblotting in human samples, accessible to most laboratories as well as by immunohistochemistry. To our knowledge, except the previously described specific polyclonal UGT2B28 antibody, also validated in UGT2B28-deficient samples (Belledant et al., 2016), it should be appreciated that the specificities of other antibodies directed against UGT2B members remain to be demonstrated and should be formally evaluated before conducting any translational studies in human samples. For instance, as highlighted in a recent study (Rouleau et al., 2016), a monoclonal anti-UGT2B10 showed significant reactivity for several UGT2B proteins, namely, the UGT2B7 protein highly 
abundant in the liver. Limitations of the current study are related to the limited sample size and the absence of ethnic groups other than white subjects. Correlation with the enzymatic activity of UGT2B17 was not performed here because of the overlapping substrate specificities of UGT2B enzymes and the lack of a known specific probe substrate for the enzyme since testosterone and etiocholanolone are conjugated by other UGTs (Green et al., 1994; Levesque et al., 1997). Based on our data, it will also be required to evaluate whether UGT2B17 expression is influenced by the rs59678213 variation in additional tissues, namely, intestine and prostate. Future research should also be directed toward finding additional molecular determinants of UGT2B17 expression, which may well be tissue-specific. Besides additional genetic variants in the UGT2B17 gene locus, those affecting expression of the FOXA1 transcription factor might also be important. This knowledge will help understand the biologic impact of this enzyme in health and diseases, namely, on the endocrine system (Juul et al., 2009; Mouritsen et al., 2018), osteoporosis (Yang et al., 2008), carcinogen exposure (Chen et al., 2010), head and neck cancer (Mafune et al., 2015), prostate cancer (Kpoghomou et al., 2013; Li et al., 2016), chronic lymphocytic leukemia (Gruber et al., 2013; Bhoi et al., 2016), pediatric cancer (Ishimaru et al., 2017), and on inactivation of antineoplastic and other therapeutic agents (Kang et al., 2010; Sun et al., 2010; Wang et al., 2012).

\section{Conclusion}

We developed a monoclonal antibody that specifically recognizes UGT2B17 in the liver, with no cross-reactivity with other UGT2B enzymes, such as UGT2B7 and UGT2B15, which are both involved in steroids and drug glucuronidation. Detection of UGT2B17 by immunoblotting revealed a high degree of variations in the liver, displaying a remarkable on or off expression pattern and was also effective for IHC experiments with liver samples. Although our work exposes key determinants of UGT2B17 expression in the liver, other significant variations remain to be explained in future studies with larger cohorts, especially in the UGT2B17 rs59678213A high-expressors subgroup. The availability of a specific monoclonal UGT2B17 antibody will help speed up discoveries to clarify mechanisms of its regulation and variability in the liver and extrahepatic tissues ultimately to appraise its influence on human diseases and drug inactivation.

\section{Authorship Contributions}

Participated in research design: Lévesque, Guillemette.

Conducted experiments: Labriet, Desjardins, Villeneuve, Hovington, Brisson, Caron, Fallon, Klein.

Contributed new reagents or analytic tools: Périgny, Têtu, Lacombe, Fallon, Klein, Smith, Zanger.

Performed data analysis: Émond, Labriet, Simonyan.

Wrote or contributed to writing of the manuscript: Émond, Rouleau, Guillemette, Lévesque.

\section{References}

Balliet RM, Chen G, Gallagher CJ, Dellinger RW, Sun D, and Lazarus P (2009) Characterization of UGTs active against SAHA and association between SAHA glucuronidation activity phenotype with UGT genotype. Cancer Res 69:2981-2989.

Beaulieu M, Lévesque E, Hum DW, and Bélanger A (1996) Isolation and characterization of a novel cDNA encoding a human UDP-glucuronosyltransferase active on C19 steroids. $J$ Biol Chem 271:22855-22862.

Beaulieu M, Lévesque E, Tchernof A, Beatty BG, Bélanger A, and Hum DW (1997) Chromosomal localization, structure, and regulation of the UGT2B17 gene, encoding a C19 steroid metabolizing enzyme. DNA Cell Biol 16:1143-1154.

Bélanger A, Hum DW, Beaulieu M, Lévesque E, Guillemette C, Tchernof A, Bélanger G, Turgeon D, and Dubois S (1998) Characterization and regulation of UDP-glucuronosyltransferases in steroid target tissues. J Steroid Biochem Mol Biol 65:301-310.

Belledant A, Hovington H, Garcia L, Caron P, Brisson H, Villeneuve L, Simonyan D, Têtu B, Fradet Y, Lacombe L, et al. (2016) The UGT2B28 sex-steroid inactivation pathway is a regulator of steroidogenesis and modifies the risk of prostate cancer progression. Eur Urol 69:601-609. Bhatt DK, Basit A, Zhang H, Gaedigk A, Lee SB, Claw KG, Mehrotra A, Chaudhry AS, Pearce RE, Gaedigk R, et al. (2018) Hepatic abundance and activity of androgen- and drug-metabolizing enzyme UGT2B17 are associated with genotype, age, and sex. Drug Metab Dispos 46:888-896.

Bhoi S, Baliakas P, Cortese D, Mattsson M, Engvall M, Smedby KE, Juliusson G, Sutton LA, and Mansouri L (2016) UGT2B17 expression: a novel prognostic marker within IGHV-mutated chronic lymphocytic leukemia? Haematologica 101:e63-e65.

Chen G, Giambrone NE Jr, Dluzen DF, Muscat JE, Berg A, Gallagher CJ, and Lazarus P (2010) Glucuronidation genotypes and nicotine metabolic phenotypes: importance of functional UGT2B10 and UGT2B 17 polymorphisms. Cancer Res 70:7543-7552.

Chouinard S, Pelletier G, Bélanger A, and Barbier O (2006) Isoform-specific regulation of uridine diphosphate-glucuronosyltransferase $2 \mathrm{~B}$ enzymes in the human prostate: differential consequences for androgen and bioactive lipid inactivation. Endocrinology 147:5431-5442.

Fallon JK, Neubert H, Hyland R, Goosen TC, and Smith PC (2013) Targeted quantitative proteomics for the analysis of 14 UGT1As and -2Bs in human liver using NanoUPLC-MS/MS with selected reaction monitoring. J Proteome Res 12:4402-4413.

Gallagher CJ, Balliet RM, Sun D, Chen G, and Lazarus P (2010) Sex differences in UDP-glucuronosyltransferase 2B17 expression and activity. Drug Metab Dispos 38:2204-2209.

Gallagher CJ, Kadlubar FF, Muscat JE, Ambrosone CB, Lang NP, and Lazarus P (2007) The UGT2B17 gene deletion polymorphism and risk of prostate cancer: a case-control study in Caucasians. Cancer Detect Prev 31:310-315.

Gomes AM, Winter S, Klein K, Turpeinen M, Schaeffeler E, Schwab M, and Zanger UM (2009) Pharmacogenomics of human liver cytochrome P450 oxidoreductase: multifactorial analysis and impact on microsomal drug oxidation. Pharmacogenomics 10:579-599.

Green MD, Oturu EM, and Tephly TR (1994) Stable expression of a human liver UDP-glucuronosyltransferase (UGT2B15) with activity toward steroid and xenobiotic substrates. Drug Metab Dispos 22:799-805.

Gruber M, Bellemare J, Hoermann G, Gleiss A, Porpaczy E, Bilban M, Le T, Zehetmayer S, Mannhalter C, Gaiger A, et al. (2013) Overexpression of uridine diphospho glucuronosyltransferase 2B17 in high-risk chronic lymphocytic leukemia. Blood 121:1175-1183.

Guillemette C, Lévesque E, Harvey M, Bellemare J, and Menard V (2010) UGT genomic diversity: beyond gene duplication. Drug Metab Rev 42:24-44

Guillemette C, Lévesque É, and Rouleau M (2014) Pharmacogenomics of human uridine diphospho-glucuronosyltransferases and clinical implications. Clin Pharmacol Ther 96:324-339. Hu DG, Gardner-Stephen D, Severi G, Gregory PA, Treloar J, Giles GG, English DR, Hopper JL, Tilley WD, and Mackenzie PI (2010) A novel polymorphism in a forkhead box A1 (FOXA1) binding site of the human UDP glucuronosyltransferase 2B17 gene modulates promoter activity and is associated with altered levels of circulating androstane- $3 \alpha, 17 \beta$-diol glucuronide. Mol Pharmacol 78:714-722.

Hum DW, Bélanger A, Lévesque E, Barbier O, Beaulieu M, Albert C, Vallée M, Guillemette C, Tchernof A, Turgeon D, et al. (1999) Characterization of UDP-glucuronosyltransferases active on steroid hormones. J Steroid Biochem Mol Biol 69:413-423.

Ishimaru S, Yuza Y, Kaneko T, and Urashima M (2017) Effect of UGT2B17 deletion polymorphism on prognosis in pediatric cancer. Pediatr Int (Roma) 59:427-431

Izukawa T, Nakajima M, Fujiwara R, Yamanaka H, Fukami T, Takamiya M, Aoki Y, Ikushiro S, Sakaki T, and Yokoi T (2009) Quantitative analysis of UDP-glucuronosyltransferase (UGT) 1A and UGT2B expression levels in human livers. Drug Metab Dispos 37:1759-1768.

Jones NR and Lazarus P (2014) UGT2B gene expression analysis in multiple tobacco carcinogentargeted tissues. Drug Metab Dispos 42:529-536.

Juul A, Sørensen K, Aksglaede L, Garn I, Rajpert-De Meyts E, Hullstein I, Hemmersbach P, and Ottesen AM (2009) A common deletion in the uridine diphosphate glucuronyltransferase (UGT) 2B17 gene is a strong determinant of androgen excretion in healthy pubertal boys. J Clin Endocrinol Metab 94:1005-1011.

Kahma H, Filppula AM, Neuvonen M, Tarkiainen EK, Tornio A, Holmberg MT, Itkonen MK, Finel M, Neuvonen PJ, Niemi M, et al. (2018) Clopidogrel carboxylic acid glucuronidation is mediated mainly by UGT2B7, UGT2B4, and UGT2B 17: implications for pharmacogenetics and drug-drug interactions. Drug Metab Dispos 46:141-150.

Kang SP, Ramirez J, House L, Zhang W, Mirkov S, Liu W, Haverfield E, and Ratain MJ (2010) A pharmacogenetic study of vorinostat glucuronidation. Pharmacogenet Genomics 20:638-641.

Kpoghomou MA, Soatiana JE, Kalembo FW, Bishwajit G, and Sheng W (2013) UGT2B17 polymorphism and risk of prostate cancer: a meta-analysis. ISRN Oncol 2013:465916.

Lazarus P, Zheng Y, Runkle EA, Muscat JE, and Wiener D (2005) Genotype-phenotype correlation between the polymorphic UGT2B17 gene deletion and NNAL glucuronidation activities in human liver microsomes. Pharmacogenet Genomics 15:769-778.

Lépine J, Audet-Walsh E, Grégoire J, Têtu B, Plante M, Ménard V, Ayotte P, Brisson J, Caron P, Villeneuve L, et al. (2010) Circulating estrogens in endometrial cancer cases and their relationship with tissular expression of key estrogen biosynthesis and metabolic pathways. $J$ Clin Endocrinol Metab 95:2689-2698.

Lévesque E, Beaulieu M, Green MD, Tephly TR, Bélanger A, and Hum DW (1997) Isolation and characterization of UGT2B15(Y85): a UDP-glucuronosyltransferase encoded by a polymorphic gene. Pharmacogenetics 7:317-325.

Li H, Xie N, Chen R, Verreault M, Fazli L, Gleave ME, Barbier O, and Dong X (2016) UGT2B17 expedites progression of castration-resistant prostate cancers by promoting ligand-independent AR signaling. Cancer Res 76:6701-6711.

Liu W, Ramírez J, Gamazon ER, Mirkov S, Chen P, Wu K, Sun C, Cox NJ, Cook E Jr, Das S, et al. (2014) Genetic factors affecting gene transcription and catalytic activity of UDPglucuronosyltransferases in human liver. Hum Mol Genet 23:5558-5569.

Mafune A, Hama T, Suda T, Suzuki Y, Ikegami M, Sakanashi C, Imai S, Nakashima A, Yokoo T, Wada K, et al. (2015) Homozygous deletions of UGT2B17 modifies effects of smoking on TP53-mutations and relapse of head and neck carcinoma. BMC Cancer 15:205.

Margaillan G, Rouleau M, Fallon JK, Caron P, Villeneuve L, Turcotte V, Smith PC, Joy MS, and Guillemette C (2015a) Quantitative profiling of human renal UDP-glucuronosyltransferases and glucuronidation activity: a comparison of normal and tumoral kidney tissues. Drug Metab Dispos 43:611-619.

Margaillan G, Rouleau M, Klein K, Fallon JK, Caron P, Villeneuve L, Smith PC, Zanger UM, and Guillemette C (2015b) Multiplexed targeted quantitative proteomics predicts hepatic glucuronidation potential. Drug Metab Dispos 43:1331-1335.

McCarroll SA, Hadnott TN, Perry GH, Sabeti PC, Zody MC, Barrett JC, Dallaire S, Gabriel SB, Lee C, Daly MJ, et al.; International HapMap Consortium (2006) Common deletion polymorphisms in the human genome. Nat Genet 38:86-92.

Ménard V, Eap O, Harvey M, Guillemette C, and Lévesque E (2009) Copy-number variations (CNVs) of the human sex steroid metabolizing genes UGT2B17 and UGT2B28 and their associations with a UGT2B15 functional polymorphism. Hum Mutat 30:1310-1319. 
Mouritsen A, Busch AS, Aksglaede L, Rajpert-De Meyts E, and Juul A (2018) Deletion in the uridine diphosphate glucuronyltransferase $2 \mathrm{~B} 17$ gene is associated with delayed pubarche in healthy boys. Endocr Connect 7:460-465.

Nadeau G, Bellemare J, Audet-Walsh É, Flageole C, Huang SP, Bao BY, Douville P, Caron P, Fradet Y, Lacombe L, et al. (2011) Deletions of the androgen-metabolizing UGT2B genes have an effect on circulating steroid levels and biochemical recurrence after radical prostatectomy in localized prostate cancer. J Clin Endocrinol Metab 96:E1550-E1557.

Oda S, Kato Y, Hatakeyama M, Iwamura A, Fukami T, Kume T, Yokoi T, and Nakajima M (2017) Evaluation of expression and glycosylation status of UGT1A10 in Supersomes and intestinal epithelial cells with a novel specific UGT1A10 monoclonal antibody. Drug Metab Dispos 45: $1027-1034$.

Oda S, Nakajima M, Hatakeyama M, Fukami T, and Yokoi T (2012) Preparation of a specific monoclonal antibody against human UDP-glucuronosyltransferase (UGT) 1 A9 and evaluation of UGT1A9 protein levels in human tissues. Drug Metab Dispos 40:1620-1627.

Rouleau M, Tourancheau A, Girard-Bock C, Villeneuve L, Vaucher J, Duperré AM, Audet-Delage Y, Gilbert I, Popa I, Droit A, et al. (2016) Divergent expression and metabolic functions of human glucuronosyltransferases through alternative splicing. Cell Rep 17:114-124.

Sakamoto A, Matsumaru T, Ishiguro N, Schaefer O, Ohtsuki S, Inoue T, Kawakami H, and Terasaki T (2011) Reliability and robustness of simultaneous absolute quantification of drug transporters, cytochrome P450 enzymes, and Udp-glucuronosyltransferases in human liver tissue by multiplexed MRM/selected reaction monitoring mode tandem mass spectrometry with nano-liquid chromatography. J Pharm Sci 100:4037-4043.

Sato Y, Nagata M, Tetsuka K, Tamura K, Miyashita A, Kawamura A, and Usui T (2014) Optimized methods for targeted peptide-based quantification of human uridine $5^{\prime}$-diphosphateglucuronosyltransferases in biological specimens using liquid chromatography-tandem mass spectrometry. Drug Metab Dispos 42:885-889.

Stingl JC, Bartels H, Viviani R, Lehmann ML, and Brockmöller J (2014) Relevance of UDP-glucuronosyltransferase polymorphisms for drug dosing: a quantitative systematic review. Pharmacol Ther 141:92-116.

Sumida A, Kinoshita K, Fukuda T, Matsuda H, Yamamoto I, Inaba T, and Azuma J (1999) Relationship between mRNA levels quantified by reverse transcription-competitive PCR and metabolic activity of CYP3A4 and CYP2E1 in human liver. Biochem Biophys Res Commun 262 499-503.

Sun D, Chen G, Dellinger RW, Sharma AK, and Lazarus P (2010) Characterization of 17-dihydroexemestane glucuronidation: potential role of the UGT2B17 deletion in exemestane pharmacogenetics. Pharmacogenet Genomics 20:575-585.
Taghavi T, St Helen G, Benowitz NL, and Tyndale RF (2017) Effect of UGT2B10, UGT2B17, FMO3, and OCT2 genetic variation on nicotine and cotinine pharmacokinetics and smoking in African Americans. Pharmacogenet Genomics 27:143-154

Tourancheau A, Margaillan G, Rouleau M, Gilbert I, Villeneuve L, Lévesque E, Droit A, and Guillemette C (2016) Unravelling the transcriptomic landscape of the major phase II UDP-glucuronosyltransferase drug metabolizing pathway using targeted RNA sequencing. Pharmacogenomics $J$ 16:60-70.

Tourancheau A, Rouleau M, Guauque-Olarte S, Villeneuve L, Gilbert I, Droit A, and Guillemette C (2018) Quantitative profiling of the UGT transcriptome in human drug-metabolizing tissues. Pharmacogenomics $J$ 18:251-261

Tremmel R, Klein K, Winter S, Schaeffeler E, and Zanger UM (2016) Gene copy number variation analysis reveals dosage-insensitive expression of CYP2E1. Pharmacogenomics $J$ 16:551-558.

Turgeon D, Carrier JS, Chouinard S, and Bélanger A (2003) Glucuronidation activity of the UGT2B17 enzyme toward xenobiotics. Drug Metab Dispos 31:670-676.

Turgeon D, Carrier JS, Lévesque E, Hum DW, and Bélanger A (2001) Relative enzymatic activity, protein stability, and tissue distribution of human steroid-metabolizing UGT2B subfamily members. Endocrinology 142:778-787.

Wang YH, Trucksis M, McElwee JJ, Wong PH, Maciolek C, Thompson CD, Prueksaritanont T, Garrett GC, Declercq R, Vets E, et al. (2012) UGT2B17 genetic polymorphisms dramatically affect the pharmacokinetics of MK-7246 in healthy subjects in a first-in-human study. Clin Pharmacol Ther 92:96-102.

Xue Y, Sun D, Daly A, Yang F, Zhou X, Zhao M, Huang N, Zerjal T, Lee C, Carter NP, et al. (2008) Adaptive evolution of UGT2B17 copy-number variation. Am J Hum Genet 83:337-346.

Yang TL, Chen XD, Guo Y, Lei SF, Wang JT, Zhou Q, Pan F, Chen Y, Zhang ZX, Dong SS, et al (2008) Genome-wide copy-number-variation study identified a susceptibility gene, UGT2B17, for osteoporosis. Am J Hum Genet 83:663-674.

Address correspondence to: Dr. Eric Lévesque, $\mathrm{CHU}$ de Québec Research Centre, R4720, 2705 Blvd. Laurier, Québec, QC G1V 4G2, Canada. E-mail: eric.levesque@crchudequebec.ulaval.ca; or Dr. Chantal Guillemette, Canada Research Chair in Pharmacogenomics, CHU de Québec Research Centre, R4720, 2705 Blvd. Laurier, Québec, QC G1V 4G2, Canada. E-mail: chantal. guillemette@crchudequebec.ulaval.ca 